\title{
On the Minimum Number of Completely 3-Scrambling Permutations
}

\author{
Jun Tarui \\ Department of Information and Communication Engineering, University of Electro-Communications \\ Chofu, Tokyo 182-8585 Japan tarui@ice. uec. ac.jp
}

\begin{abstract}
A family $\mathcal{P}=\left\{\pi_{1}, \ldots, \pi_{q}\right\}$ of permutations of $[n]=\{1, \ldots, n\}$ is completely $k$-scrambling [Spencer, 1972; Füredi, 1996] if for any distinct $k$ points $x_{1}, \ldots, x_{k} \in[n]$, permutations $\pi_{i}$ 's in $\mathcal{P}$ produce all $k$ ! possible orders on $\pi_{i}\left(x_{1}\right), \ldots, \pi_{i}\left(x_{k}\right)$. Let $N^{*}(n, k)$ be the minimum size of such a family. This paper focuses on the case $k=3$. By a simple explicit construction, we show the following upper bound, which we express together with the lower bound due to Füredi for comparison.
\end{abstract}

$$
\frac{2}{\log _{2} e} \log _{2} n \leq N^{*}(n, 3) \leq 2 \log _{2} n+(1+o(1)) \log _{2} \log _{2} n
$$

We also prove the existence of $\lim _{n \rightarrow \infty} N^{*}(n, 3) / \log _{2} n=c_{3}$. Determining the value $c_{3}$ and proving the existence of $\lim _{n \rightarrow \infty} N^{*}(n, k) / \log _{2} n=c_{k}$ for $k \geq 4$ remain open.

\section{Introduction and Summary}

Following Spencer [Sp72] and Füredi [Fü96], call a family $\mathcal{P}=\left\{\pi_{1}, \ldots, \pi_{q}\right\}$ of permutations of $[n]$ completely $k$-scrambling if for any distinct $x_{1}, x_{2}, \ldots, x_{k} \in[n]$, there exists a permutation $\pi_{i} \in \mathcal{P}$ such that $\pi_{i}\left(x_{1}\right)<\pi_{i}\left(x_{2}\right)<\cdots<\pi_{i}\left(x_{k}\right)$; or equivalently, $\pi_{i}$ 's applied to $x_{1}, x_{2}, \ldots, x_{k}$ produce all $k$ ! orders. This paper focuses on the case $k=3$. Following Füredi [Fü96], say that a family $\mathcal{P}$ is 3 -mixing if for any distinct $x, y, z \in[n]$, there is a permutation $\pi_{i} \in \mathcal{P}$ that places $x$ between $y$ and $z$, i.e., there is a permutation $\pi_{i}$ such that either $\pi_{i}(y)<\pi_{i}(x)<\pi_{i}(z)$ or $\pi_{i}(z)<\pi_{i}(x)<\pi_{i}(y)$.

Let $N^{*}(n, k)$ be the minimum $q$ such that completely $k$-scrambling $q$ permutations exist for $[n]$. The best known bounds for $N^{*}(n, k)$ can be expressed as follows. For arbitrary fixed $k \geq 3$, as $n \rightarrow \infty$,

$$
\left(\frac{1}{\log _{2} e}(k-1) !+o(1)\right) \log _{2} n \leq N^{*}(n, k) \leq \frac{k}{\log _{2}(k ! /(k !-1))} \log _{2} n .
$$

The coefficient of the upper bound in (1) is $\Theta(k \cdot k !)$; thus the gap between the coefficients of the lower and upper bounds in (1) is $\Theta\left(k^{2}\right)$. The upper bound in (1) was shown by Spencer [Sp72] by a probabilistic argument, where one considers the probability that some order among some $x_{1}, \ldots, x_{k}$ is never produced by $q$ independent random permutations. The lower bound in (1) was first proved by Füredi [Fü96] for $k=3$, and was proved for $k \geq 3$ by Radhakrishnan [Ra03]; entropy arguments are used in both work; 
the factor $\log _{2} e$ in the lower bound comes from the fact that $\int_{0}^{1} H(x) d x=\left(\log _{2} e\right) / 2$, where $H(x)$ is the binary entropy function.

As for the case $k=3$, Füredi [Fü96] has shown that

$$
\frac{2}{\log _{2} e} \log _{2} n \leq N^{*}(n, 3) \leq\left(\frac{10}{\log _{2} 7}\right) \log _{2} n+O(1),
$$

where the coefficients of $\log _{2} n$ are $1.38 \ldots$ and $3.56 \ldots$ in (2). The lower bound in (2) is in fact a lower bound for the case where we only require a family to be 3-mixing. No better lower bound for completely 3 -scrambling families is known. If a family $\mathcal{P}=\left\{\pi_{1}, \ldots, \pi_{q}\right\}$ is 3-mixing, by adding to $\mathcal{P}$ the $q$ reverse permutations of $\pi_{i}$ 's mapping $x \mapsto n+1-\pi_{i}(x)$, we can obtain completely 3 -scrambling $2 q$ permutations. Ishigami [Is95] has given an efficient recursive construction of 3-mixing families starting with a 3-mixing family of five permutations of $\{1, \ldots, 7\}$. Füredi [Fü96] gave the upper bound in (2) by making these observations and doubling the size of Ishigami's 3-mixing family.

In this paper, we first give an improved upper bound for $N^{*}(n, 3)$ by a simple construction. Let $f(q)$ be the maximum $n$ such that completely 3 -scrambling $q$ permutations exist for $[n]$.

\section{Theorem 1}

$$
f(q) \geq\left(\begin{array}{l}
\lfloor q / 2\rfloor \\
\lfloor q / 4\rfloor
\end{array}\right)
$$

The following upper bound on $N^{*}(n, 3)$ readily follows.

\section{Corollary 1}

$$
N^{*}(n, 3) \leq 2 \log _{2} n+(1+o(1)) \log _{2} \log _{2} n
$$

It seems natural to conjecture that for every fixed $k \geq 3$, as $n \rightarrow \infty, N^{*}(n, k)=\left(c_{k}+o(1)\right) \log _{2} n$ for some $c_{k}$. We show the existence of limit for the case $k=3$ :

\section{Theorem 2}

$$
\lim _{q \rightarrow \infty} \frac{\log _{2} f(q)}{q}=C \text { exists. }
$$

The following immediately follows.

\section{Corollary 2}

$$
\lim _{n \rightarrow \infty} \frac{N^{*}(n, 3)}{\log _{2} n}=1 / C=c_{3} \text { exists. }
$$




\section{Proofs}

We can identify in a natural way a total order $\phi$ on $[n]$ and the permutation of $[n]$ induced by $\phi$; thus we speak interchangeably in terms of permutations and total orders. In fact for an arbitrary finite set $U$ with $n$ elements, we can assume for our purposes that $U$ is identified with $[n]$ in an arbitrary fixed way, and speak about permutations of $U$ in terms of total orders on $U$.

Proof of Theorem 1. Put $r=\lfloor q / 2\rfloor$ and let $\mathcal{F}=\left\{A_{1}, A_{2}, \ldots, A_{m}\right\}$ be a family of subsets of $\{1, \ldots, r\}$ such that $A_{i} \nsubseteq A_{j}$ for all $i \neq j$; i.e., $\mathcal{F}$ is an antichain.

For each point $x \in\{1, \ldots, r\}$, define two orders $\phi_{x}$ and $\psi_{x}$ on $\mathcal{F}$. In both orders $\phi_{x}$ and $\psi_{x}$, the sets $A_{i}$ containing the point $x$ are smaller than all the sets $A_{k}$ not containing $x$. Among the sets containing $x$ and among the sets not containing $x$ : in the order $\phi_{x}, A_{i}<A_{j}$ precisely when $i<j$; in the order $\psi_{x}$, this is reversed, and $A_{i}<A_{j}$ precisely when $i>j$.

We claim that for arbitrary distinct $i, j, k \in[m]$, there exists an order $\theta \in\left\{\phi_{1}, \psi_{1}, \phi_{2}, \psi_{2}, \ldots, \phi_{r}, \psi_{r}\right\}$ such that $A_{i}<A_{j}<A_{k}$ in the order $\theta$. To see the claim fix a point $x \in\left(A_{i}-A_{k}\right) \neq \emptyset$, i.e., $x \in A_{i}$ and $x \notin A_{k}$. Depending on whether $x \in A_{j}$ or $x \notin A_{j}$, we specify an order $\theta$ that produces the ordering $A_{i}<A_{j}<A_{k}$.

Case $x \in A_{j}$ : Let $\theta=\phi_{x}$ if $i<j$ and let $\theta=\psi_{x}$ if $i>j$.

Case $x \notin A_{j}$ : Let $\theta=\phi_{x}$ if $j<k$ and let $\theta=\psi_{x}$ if $j>k$.

Clearly under the order $\theta, A_{i}<A_{j}<A_{k}$. Hence the $2 r$ orders thus defined on $[m]$ are completely 3 -scrambling. We obtain the theorem by taking $\mathcal{F}$ to be the family of all subsets of $\{1, \ldots, r\}$ with cardinality $\lfloor r / 2\rfloor=\lfloor q / 4\rfloor$.

Proof of Theorem 2. Our proof of Theorem 2 will be basically similar to Füredi's proof [Fü96] of the existence of $\lim _{q \rightarrow \infty}\left(\log _{2} g(q)\right) / q$, where $g(q)$ is the maximum $n$ such that 3-mixing $q$ permutations exist for $[n]$. To make a recursive construction go through for scrambling permutations, we introduce and use red-blue colored doubly reversing permutations: Call a family $\mathcal{P}=\left\{\pi_{1}, \ldots, \pi_{q}\right\}$ of permutations of $[n]$ 2-reversing if there is a coloring $\chi:\left\{\pi_{1}, \ldots, \pi_{q}\right\} \rightarrow\{$ red, blue $\}$ such that for every distinct $i, j \in[n]$, there are red $\pi_{\kappa}$, red $\pi_{\lambda}$, blue $\pi_{\mu}$, and blue $\pi_{\nu}$ satisfying

$$
\pi_{\kappa}(i)<\pi_{\kappa}(j), \quad \pi_{\lambda}(i)>\pi_{\lambda}(j) ; \quad \pi_{\mu}(i)<\pi_{\mu}(j), \pi_{\nu}(i)>\pi_{\nu}(j)
$$

For a permutation $\pi$ of $[n]$, let reverse $(\pi)$ be the permutation of $[n]$ mapping $x \mapsto n+1-\pi(x)$. Let $\mathcal{P}$ be a family of permutations of $[n]$ with $|\mathcal{P}| \geq 3$. We can easily transform $\mathcal{P}$ to a 2-reversing family by adding at most two permutations as follows. Arbitrarily fix two distinct permutations $\sigma, \tau \in \mathcal{P}$ such that $\tau \neq$ reverse $(\sigma)$; such $\sigma$ and $\tau$ exist since $|\mathcal{P}| \geq 3$; add reverse $(\sigma)$ and reverse $(\tau)$ to $\mathcal{P}$; color $\sigma$ and reverse $(\sigma)$ red; color $\tau$ and reverse $(\tau)$ blue; color the remaining permutations arbitrarily.

Let $f^{*}(q)$ be the maximum $n$ such that completely 3-scrambling and 2-reversing $q$ permutations exist for $[n]$. By definition and from the discussion above we have

$$
f^{*}(q) \leq f(q) \leq f^{*}(q+2)
$$

\section{Claim 1}

$$
f^{*}(q+r) \geq f^{*}(q) f^{*}(r)
$$


For the moment we assume that Claim 1 holds and go on to derive Theorem 2.

The sequence $(1 / q) \log _{2} f^{*}(q)$ is bounded above. ¿From this and Claim 1 it follows by classical calculus (Fekete's theorem) that

$$
\lim _{q \rightarrow \infty} \frac{1}{q} \log _{2} f^{*}(q)=\limsup _{q \rightarrow \infty} \frac{1}{q} \log _{2} f^{*}(q) .
$$

From (3) it now follows that

$$
\lim _{q \rightarrow \infty} \frac{1}{q} \log _{2} f(q)=\lim _{q \rightarrow \infty} \frac{1}{q} \log _{2} f^{*}(q)
$$

Thus we are left to prove Claim 1 .

Let $\mathcal{S}=\left\{\sigma_{1}, \ldots, \sigma_{q}\right\}$ and $\mathcal{T}=\left\{\tau_{1}, \ldots, \tau_{r}\right\}$ be completely 3 -scrambling and 2-reversing families of permutations of $[l]$ and $[m]$ respectively. Assume that both families are validly red-blue colored. Let $U=\{(i, j): 1 \leq i \leq l, 1 \leq j \leq m\}$; think of $U$ as a matrix with $l$ rows and $m$ columns. We will show that we can define $q+r$ orders on $U$ that are completely 3 -scrambling and 2-reversing. Note that from this Claim 1 follows.

Let $x=(i, j)$ and $y=\left(i^{\prime}, j^{\prime}\right)$ be distinct elements of $U$. For $k=1, \ldots, q$, define the order $\tilde{\sigma}_{k}$ using $\sigma_{k}$ in a row-major form as follows: if $i \neq i^{\prime}$, order $x$ and $y$ according to the order of $\sigma_{k}(i)$ and $\sigma_{k}\left(i^{\prime}\right)$. When $i=i^{\prime}$ : if $\sigma_{k}$ is red, $(i, j)<\left(i, j^{\prime}\right) \Longleftrightarrow j<j^{\prime}$; if $\sigma_{k}$ is blue, $(i, j)<\left(i, j^{\prime}\right) \Longleftrightarrow j>j^{\prime}$. Similarly for $k=1, \ldots, r$, define the order $\tilde{\tau}_{k}$ on $U$ in a column-major form: when $j \neq j^{\prime}, x<y \Longleftrightarrow \tau_{k}(j)<\tau_{k}\left(j^{\prime}\right)$; when $j=j^{\prime}$ : if $\tau_{k}$ is red, $(i, j)<\left(i^{\prime}, j\right) \Longleftrightarrow i<i^{\prime}$; if $\tau_{k}$ is blue, $(i, j)<\left(i^{\prime}, j\right) \Longleftrightarrow i>i^{\prime}$. As for colors, let $\tilde{\sigma}_{k}$ and $\tilde{\tau}_{k}$ inherit the colors of $\sigma_{k}$ and $\tau_{k}$.

Claim 2 The family $\mathcal{F}=\left\{\tilde{\sigma}_{1}, \ldots, \tilde{\sigma}_{q}, \tilde{\tau}_{1}, \ldots, \tilde{\tau}_{r}\right\}$ is completely 3 -scrambling and 2-reversing.

To see Claim 2, let $x_{1}=\left(i_{1}, j_{1}\right), x_{2}=\left(i_{2}, j_{2}\right), x_{3}=\left(i_{3}, j_{3}\right)$ be distinct elements of $U$. If $i_{1}, i_{2}, i_{3}$ are all distinct, $\sigma_{k}$ 's produce all six orderings of $i_{1}, i_{2}, i_{3}$, and hence $\tilde{\sigma}_{k}$ 's produce all six orderings of $x_{1}, x_{2}, x_{3}$. Similar arguments with $\tau_{k}$ 's and $\tilde{\tau}_{k}$ 's apply for the case when $j_{1}, j_{2}, j_{3}$ are all distinct.

The remaining case is when $\left|\left\{i_{1}, i_{2}, i_{3}\right\}\right|=\left|\left\{j_{1}, j_{2}, j_{3}\right\}\right|=2$. We write, e.g., 231 to express the ordering $x_{2}<x_{3}<x_{1}$. Assume that

$$
x_{1}=(i, j), x_{2}=\left(i, j^{\prime}\right), x_{3}=\left(i^{\prime}, j\right), i \neq i^{\prime}, j \neq j^{\prime} .
$$

We will see that all six orderings of $x_{1}, x_{2}, x_{3}$ are produced by checking that (1) all the four orders in which $x_{3}$ is smallest or largest, i.e., 312, 321, 123, 213 are produced and that (2) all the four orders in which $x_{2}$ is smallest or largest are produced.

A red $\tilde{\sigma}_{\kappa}$ and a blue $\tilde{\sigma}_{\mu}$ satisfying $\sigma_{\kappa}(i)<\sigma_{\kappa}\left(i^{\prime}\right)$ and $\sigma_{\mu}(i)<\sigma_{\mu}\left(i^{\prime}\right)$ produce 123 and 213 respectively. Similarly, a red $\tilde{\sigma}_{\lambda}$ and a blue $\tilde{\sigma}_{\nu}$ satisfying $\sigma_{\lambda}(i)>\sigma_{\lambda}\left(i^{\prime}\right)$ and $\sigma_{\nu}(i)>\sigma_{\nu}\left(i^{\prime}\right)$ produce 312 and 321 respectively. Thus all the four orders in which $x_{3}$ is smallest or largest are produced. Similarly, two red $\tilde{\tau}$ 's and two blue $\tilde{\tau}$ 's ordering $j$ and $j^{\prime}$ in both directions produce the four orders in which $x_{2}$ is smallest or largest.

Finally, if $x=(i, j)$ and $y=\left(i^{\prime}, j^{\prime}\right)$ are distinct points in $U$, either (i) $i \neq i^{\prime}$ or (ii) $j \neq j^{\prime}$. The 2-reversing condition is satisfied by $\tilde{\sigma}_{k}$ 's in case (i) and by $\tilde{\tau}_{k}$ 's in case (ii). 


\section{Acknowledgements}

The author thanks the anonymous referees for helpful comments.

\section{References}

[Fü96] Z. Füredi. Scrambling Permutations and Entropy of Hypergraphs, Random Structures and Algorithms, vol. 8, no. 2, pp. 97-104, 1996.

[Is95] Y. Ishigami. Containment Problems in High-Dimensional Spaces, Graphs and Combinatorics, vol. 11, pp. 327-335, 1995.

[Ra03] J. Radhakrishnan. A Note on Scrambling Permutations, Random Structures and Algorithms, vol. 22 , no. 4, pp. 435-439, 2003.

[Sp72] J. Spencer. Minimal Scrambling Sets of Simple Orders, Acta Mathematica Hungarica, vol. 22, pp. 349-353, 1972. 
